\title{
The Immunologic Injury Composite with Balloon Injury Leads to Dyslipidemia: A Robust Rabbit Model of Human Atherosclerosis and Vulnerable Plaque
}

\author{
Guangyin Zhang, ${ }^{1}$ Ming Li, ${ }^{1}$ Liangjun Li, ${ }^{1}$ Yingzhi Xu, ${ }^{1}$ Peng Li, ${ }^{1}$ Cui Yang, \\ Yanan Zhou, ${ }^{1}$ and Junping Zhang ${ }^{1,2}$ \\ ${ }^{1}$ Office building, Rm 309, First Teaching Hospital of Tianjin University of TCM, No. 314, An Shan Xi Road, Nan Kai District, \\ Tianjin 300193, China \\ ${ }^{2}$ Tianjin University of Traditional Chinese Medicine, Tianjin, China
}

Correspondence should be addressed to Junping Zhang, tjzhtcm@163.com

Received 21 March 2012; Revised 25 July 2012; Accepted 30 July 2012

Academic Editor: Renata Gorjão

Copyright ( 2012 Guangyin Zhang et al. This is an open access article distributed under the Creative Commons Attribution License, which permits unrestricted use, distribution, and reproduction in any medium, provided the original work is properly cited.

\begin{abstract}
Atherosclerosis is a condition in which a lipid deposition, thrombus formation, immune cell infiltration, and a chronic inflammatory response, but its systemic study has been hampered by the lack of suitable animal models, especially in herbalism fields. We have tried to perform a perfect animal model that completely replicates the stages of human atherosclerosis. This is the first combined study about the immunologic injury and balloon injury based on the cholesterol diet. In this study, we developed a modified protocol of the white rabbit model that could represent a novel approach to studying human atherosclerosis and vulnerable plaque.
\end{abstract}

\section{Introduction}

Atherosclerosis is the most common pathological process that leads to cardiovascular diseases (CVDs), a disease of large- and medium-sized arteries, that is characterized by a formation of atherosclerotic plaques consisting of necrotic cores, calcified regions, accumulated modified lipids, inflamed smooth muscle cells (SMCs), endothelial cells (ECs), leukocytes, and foam cells $[1,2]$. Several features of atherosclerotic plaques illustrate that atherosclerosis is a complex disease, and many components of the vascular, metabolic, and immune systems are involved in this process [3]. Although dyslipidemia like higher low-density lipoprotein (LDL) or higher triglyceride (TC) remains the most important risk factor for atherosclerosis, immune and inflammatory mechanisms of atherosclerosis have gained tremendous interest in the past 20 years $[4,5]$.

The area of atherosclerotic plaques research is day by day expanding and the animal models play a very crucial role in this onward journey. An animal model is a nonhuman animal that has a disease or injury that is similar to a human condition [6]. They are due to focal accumulation of cells within the intima of the artery [7], both intra- and extracellular lipids [8], fibrous tissues [9], complex proteoglycans, and mineral blood and blood products [10]. Even though there is no one perfect animal model that completely replicates the stages of human atherosclerosis, cholesterol feeding and mechanical endothelial injury are two common features shared by most models of atherosclerosis [11, 12]. Several characteristics of the rabbit make it an excellent model for the study of atherosclerosis. Previous experimental models for studying this vessel disease consist of rabbits and rats undergoing cholesterol feeding and mechanical endothelial injury [13]. We have built a model based on rabbit that including cholesterol feeding, immunologic injury, and mechanical endothelial injury. Studies in these models have primarily focused on the rules of changes in blood lipids and the morphology and inflammation of the arterial walls [14].

The aim of this experimental study was to evaluate the applicability of a modified white rabbit model that 
were injured with immunologic and balloon base on the cholesterol diet. We evaluated the model from cholesterol metabolism, immune and inflammatory mechanisms and impaired level of reactive oxygen species. The model that we characterize here would be useful for studying the entire process of human atherosclerosis from dysfunction of cholesterol metabolism to the formation of atherosclerotic plaques.

\section{Materials and Methods}

2.1. Animals and Experimental Design. All animal experiments were performed with the approval of the Animal Care Committee of the Tianjin University of Traditional Chinese Medicine and complied with the Animal Management Rule of the Ministry of Public Health, People's Republic of China (Documentation 55, 2001). Twenty-four, adult (3 months old, $2.0 \pm 0.2 \mathrm{~kg}$ ), male, Japanese white rabbits were purchased from Vital River Lab Animal Technology Co., Ltd. (Beijing, China) and were housed in an animal room maintained at $22 \pm 2{ }^{\circ} \mathrm{C}$ with $40 \%$ to $60 \% \mathrm{RH}$ and a light period from 8:00 to 20:00 in the Laboratory Animal Center of Tianjin university of TCM. The rabbits were divided randomly into two groups, normal-diet group (normal, $n=$ 8 ) was fed the rabbit standard diet ( $100 \mathrm{~g}$ per rabbit per day) and experimental model group (CIB, $n=16$ ) were fed an atherogenic diet (1\% cholesterol, $5 \%$ yolk, 5\% lard, and $89 \%$ standard diet). All the animals had free access to water. A scheme of the design of the atherosclerotic model study is shown in Figure 1.

2.2. Immunologic Injury and Balloon Injury. Initially, all experiment rabbits were fed with cholesterol diet (Yingbo, Tianjin, China) 2 weeks prior to injecting solcoseryl albumin (Sangon, Shanghai, China, $250 \mathrm{mg} / \mathrm{kg}$ ) from the marginal ear vein and 4 weeks after balloon injury of the abdominal aorta, followed by 6 weeks of cholesterol chow diet constantly. All rabbits underwent balloon-induced endothelial injury in the abdominal aorta after being anesthetized with 3\% pentobarbital sodium salt (Sigma-Aldrich P3761, US, $30 \mathrm{mg} / \mathrm{kg}$ body weight, iv), and balloon injury of the abdominal aortic wall was performed using a $4 \mathrm{~F}$ Fogarty catheter (Shangyikangge, Shanghai, China) introduced through a right femoral artery cutdown. After the catheter was advanced to the diaphragm, the balloon was inflated and the catheter was gently retracted toward the iliofemoral artery. This was repeated three times. The method was demonstrated in previous reports $[15,16]$.

2.3. Biochemical Parameters. Animals were bled from the marginal ear vein at baseline at the end of weeks 3, 6, and 10 ( $3 \mathrm{~mL}$ each time). Blood samples were centrifuged at $2000 \mathrm{~g}$ for 20 minutes to obtain serum and plasma. The serum was used for biomarkers assay.

Collected serum was analyzed in an automatic blood chemical analyzer (Humalyzer 2000, Nairobi, Kenya), and the serum concentrations of total cholesterol (TC), triglyceride (TG), high-density lipoprotein cholesterol (HDLC), and low-density lipoprotein cholesterol (LDL-C) were

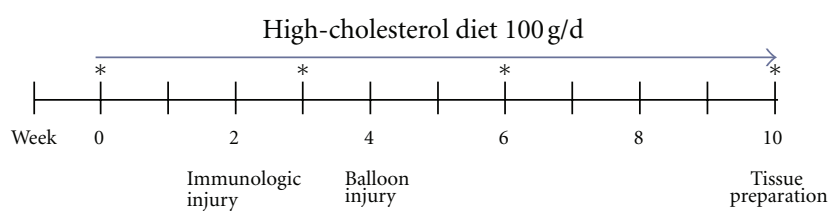

Figure 1: Schematic diagram of study timeline. Immunologic injury is followed by 2 weeks of high cholesterol feeding and balloon injury on week 4 . ${ }^{*}$ Means the times of serum collection and animal body weight.

obtained (enzyme-colorimetric method). At the same time, serum levels of superoxide dismutase (SOD), malondialdehyde (MDA), and nitric oxide (NO) were estimated by enzyme colorimetric method with kits from, Jiancheng Bioengineering Institute (Nanjing, China), and serum levels of monocyte chemoattractant protein-1 (MCP-1), tumor necrosis factor- $\alpha$ (TNF- $\alpha$ ), and oxidized LDL cholesterol (oxLDL) were measured by use of enzyme-linked immunosorbent assay (ELISA) kits (Rapid Bio, California, US).

2.4. Tissue Preparation. The experiment was continued till 10 weeks. After euthanasia of the animals by intravenous injection of pentobarbital (Sigma-Aldrich P3761, US, $50 \mathrm{mg} / \mathrm{kg}$ body weight, iv) at the end of weeks 10, the blood sample was taken via the marginal ear vein. The aorta from the aortic valve to the femoral bifurcation tissue of animals was removed. The abdominal aortic tissue was collected for histological study and remainders stored at $-80^{\circ} \mathrm{C}$ for other studies.

The abdominal aortas from the rabbits were immediately excised and cut into 10 serial $2.5 \mathrm{~mm}$ sections, with alternative sections embedded in paraffin and catalogued. The tissue sections ( $6 \mu \mathrm{m}$ thick) were cut from paraffinembedded blocks on a microtome and mounted from warm water $\left(40^{\circ} \mathrm{C}\right)$ onto adhesive microscope slides. Sections were allowed to dry overnight at room temperature and for later general histological staining, as described previously $[17,18]$.

\subsection{Histological and Morphometric Evaluation}

2.5.1. Identification of Atherosclerotic Plaques. The fatty streak lesions of the thoracic aorta in each group were easily identified by staining with Sudan III (Sangon, Shanghai, China), as described above [19]. After formalin fixation of the arteries, paraffin blocks were prepared by the following routine histological procedures. The tissue sections were obtained from the prepared paraffin blocks and were stained with hematoxylin and eosin $(\mathrm{H} \& \mathrm{E})$. All slides were examined by Olympus BX 40 Light Microscope (Olympus Corporation, Tokyo, Japan), and Champion HMIAS-2000 Image Analysis System (Wuhan Champion Image Technology Co., Ltd, Hubei, China) was used for image processing. The luminal intimal and medial cross-sectional areas of the arteries were measured and the index defined as intimal/medial area ratio was calculated from these measurements. The fibrous cap thickness was measured by drawing lines perpendicular to the lumen at three different locations of the fibrous cap 
TABLE 1: Body weight in the two groups $(\mathrm{kg})^{*}$.

\begin{tabular}{lcccrr}
\hline Groups & Number of rabbits & 0 wk (baseline) & 3 wk & 6 wk & 10 wk \\
\hline Normal & 6 & $2.11 \pm 0.20$ & $2.250 \pm 0.175$ & $2.400 \pm 0.167$ & $2.73 \pm 0.31$ \\
CIB & 10 & $2.12 \pm 0.14$ & $2.257 \pm 0.183$ & $2.242 \pm 0.156$ & $2.57 \pm 0.16$ \\
\hline
\end{tabular}

${ }^{*}$ Data are given as the mean \pm SD. No significant difference was found between the normal group and the CIB group.

TABLE 2: Lipid profile of serum in the two groups ( $\mathrm{mmol} / \mathrm{L}$ ).

\begin{tabular}{lcccccc}
\hline & Groups & $n$ & $0 \mathrm{wk}$ & $3 \mathrm{wk}$ & $6 \mathrm{wk}$ & $10 \mathrm{wk}$ \\
\hline \multirow{2}{*}{ TC } & Normal & 6 & $1.04 \pm 0.13$ & $1.43 \pm 0.40$ & $1.31 \pm 0.17$ & $1.35 \pm 0.34$ \\
& CIB & 10 & $1.08 \pm 0.40$ & $10.38 \pm 4.99^{* *}$ & $10.99 \pm 3.89^{* *}$ & $24.04 \pm 4.73^{* *}$ \\
\hline \multirow{2}{*}{ TG } & Normal & 6 & $1.14 \pm 0.39$ & $0.69 \pm 0.07$ & $0.96 \pm 0.34$ & $0.77 \pm 0.20$ \\
& CIB & 10 & $1.19 \pm 0.54$ & $1.25 \pm 0.57^{*}$ & $0.95 \pm 0.47$ & $1.42 \pm 0.51^{*}$ \\
\hline \multirow{2}{*}{ LDL-C } & Normal & 6 & $0.53 \pm 0.17$ & $0.39 \pm 0.13$ & $0.35 \pm 0.16$ & $0.67 \pm 0.21$ \\
& CIB & 10 & $0.51 \pm 0.12$ & $3.50 \pm 1.74^{* *}$ & $2.39 \pm 1.17^{* *}$ & $11.73 \pm 3.09^{* *}$ \\
\hline \multirow{2}{*}{ HDL-C } & Normal & 6 & $0.66 \pm 0.18$ & $0.68 \pm 0.09$ & $0.53 \pm 0.08$ & $0.37 \pm 0.09$ \\
& CIB & 10 & $0.60 \pm 0.15$ & $1.54 \pm 0.59^{* *}$ & $1.59 \pm 0.38^{* *}$ & $4.00 \pm 0.90^{* *}$ \\
\hline
\end{tabular}

TC: total cholesterol; TG: triglyceride; LDL-C: low-density lipoprotein cholesterol; HDL-C: high-density lipoprotein cholesterol. Data are expressed as mean \pm $\mathrm{SD},{ }^{*} P<0.05 ;{ }^{*} P<0.01$ compared with normal.

on sections stained with the measurement as well, and the mean value was calculated. The other segment was used for cryosections and cut into $6 \mu \mathrm{m}$ thick sections for Masson's trichromatic and Oil Red $\mathrm{O}$ staining, and the methods used here were demonstrated in previous reports [20,21].

2.5.2. Immunohistochemistry in Abdominal Aortas Lesions. Immunohistochemical staining involved several standard techniques as described previously [22]. In brief, all tissue sections were rinsed with $\mathrm{PBS}$ at $\mathrm{pH} 7.4$; in order to block endogenous peroxidase activity; the sections were incubated in $0.3 \% \mathrm{H}_{2} \mathrm{O}_{2}$ for $30 \mathrm{~min}$, and then washed with PBS. Followed by incubating in secondary antibody for 30 minutes at $37^{\circ} \mathrm{C}$. The secondary antibody and all subsequent reagents were diluted in PBS containing $0.1 \%$ bovine serum albumin; $200 \mu \mathrm{L}$ of the diluted solution was added to each slide and incubated in a moisture chamber. Immunohisto-chemical staining was visualized by use of a diaminobenzidine kit (TBD Science, Tianjin, China) according to the manufacturer's instructions. The primary antibodies included $\alpha$-smooth muscle cell actin (Thermo Fisher Scientific, US), anti-CD68 polyclonal antibody, antiCD31 polyclonal antibody (R\&D Systems, Minneapolis, MN, USA) matrix metalloproteinase- 9 antibody (Abcam, Cambridge, UK), vascular endothelial growth factor antibody (Santa Cruz Biotechnology, CA, USA), and NF-kB P65 subunit antibody (Thermo Fisher Scientific, USA).

\section{Statistical Analysis}

The Statistical Package for the Social Sciences (SPSS) 11.0 was used for the analysis. Results are given as mean \pm SD. Data were analyzed statistically using one-way ANOVA test followed by LSD posttest, and pairwise multiple comparisons were performed using LSD posttest. In all instances, $P$ value less than 0.05 or 0.01 was considered significant.

\section{Results}

4.1. Design of the Animal Experiments. During the experiment, six rabbits in the CIB group and two rabbits in the normal group died of anesthesia overdose or diarrhea. Data was available for analysis for 6 rabbits in the normal group and 10 for CIB group. The animal body weight was not statistically significant in the CIB group compared with the normal group in week $0,3,6,10$, as shown in Table 1 . We found that the animal body weight of CIB group was slightly decreased at the end of the sixth weekend it was probably due more to diet reduction after surgery.

4.2. Cholesterol Levels. Table 2 shows lipid profile of serum in two groups; the levels of serum TC, TG, LDL-C, and HDL-C were elevated in general. Serum TC levels in the CIB group were higher than those in the control group at 3rd, 6 th, and 10th weekend, respectively $(P<0.01)$. Compared with the normal group, serum TG levels were increased in the CIB group at the 3rd weekend $(P<0.05)$, and the levels significantly increased at the 10 th weekend $(P<0.05)$. However, which levels in CIB group not increased like TC than in the control group at the 6th weekend. Under a high cholesterol diet, the levels of the serum LDL-C of the rabbits were elevated as well. The serum LDL-C levels in the CIB group were higher than those in the normal group at the $3 \mathrm{rd}$, 6th weeks, respectively, and showed a significant difference at the 10th weekend. Serum HDL-C levels kept the same trend in both groups.

\subsection{Histological and Morphometric Evaluation}

4.3.1. Identification of Atherosclerotic Plaques. The fatty streak lesions of the aorta in two groups were easily identified by staining with Sudan III or by macroscopic observation. Broad and fused fatty streak lesions were easily found in CIB 

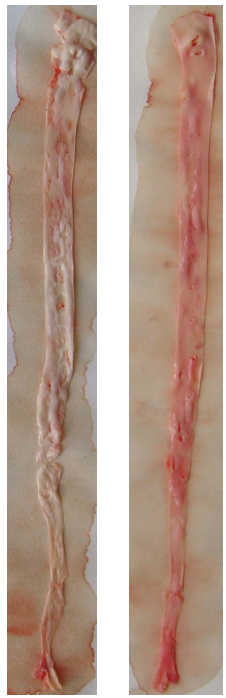

(a)

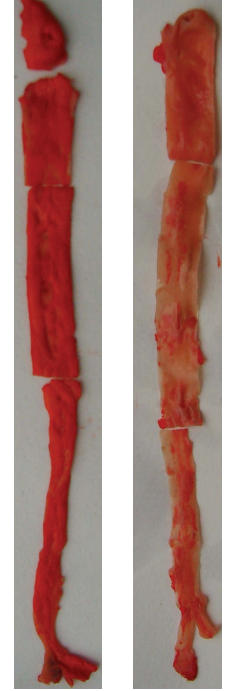

(b)

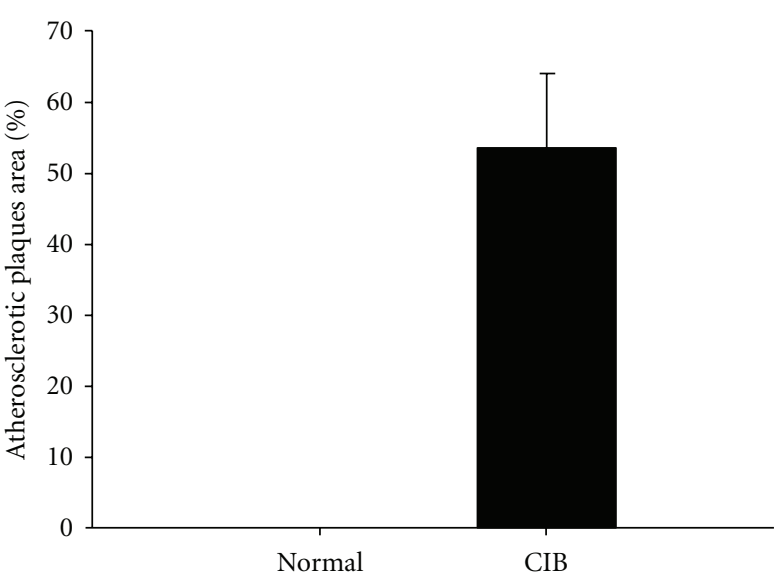

(c)

Figure 2: Macroscopic observation of atherosclerotic plaques area and staining with Sudan III. (a) Macroscopic observation of the whole aorta. (b) Representative en face Sudan staining of the whole aorta. (c) Percentage of the atherosclerotic plaques area in both groups. The Ratio of atherosclerotic plaques area to the whole aorta was $54 \pm 11 \%$ in CIB group, but no atherosclerotic plaques area has been detected in the normal group.

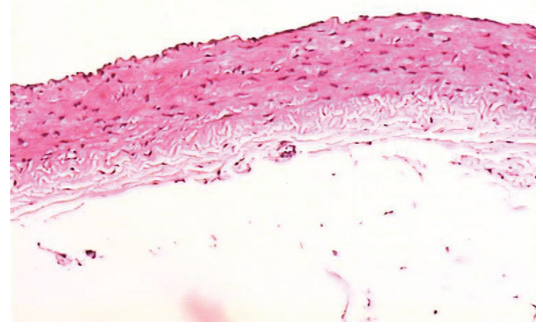

(a)



(d)

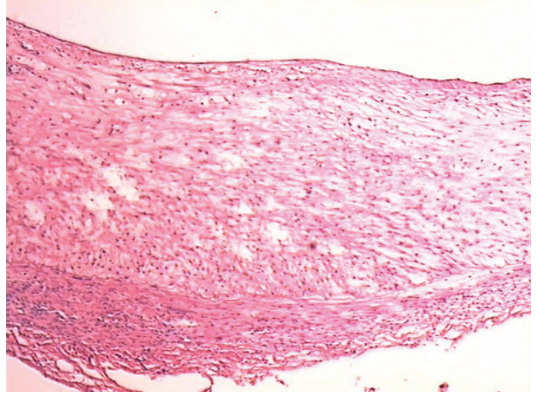

(b)

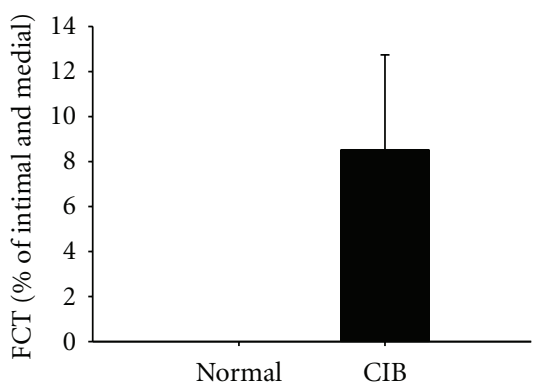

(e)

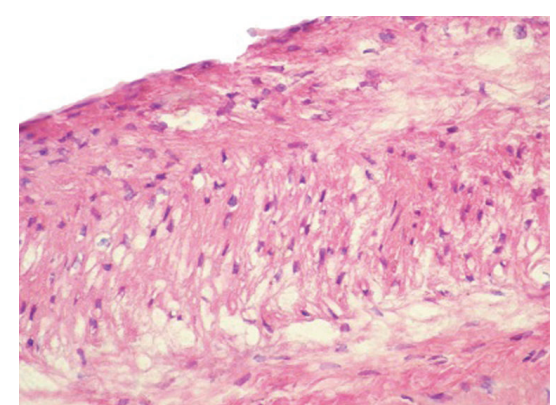

(c)



(f)

FIgURE 3: Histological examples of lipid core and the fibrous cap associated with rabbit atherosclerotic plaques. (a) A cross section of a normal rabbit aorta. (b) A cross section of a CIB rabbit atherosclerotic plaques. (c) Higher magnification of the atherosclerotic plaques site shows a thin fibrous cap. The fibrous cap thickness, (d) ratio of fibrous cap to intima media thickness (e), and vulnerability index (f) were calculated. Data are expressed as mean $\pm \mathrm{SD},{ }^{* *} P<0.01$ compared with normal.

group that was stained most seriously, almost the whole aorta was red, whereas no atherosclerotic plaques were observed in the normal group (Figures 2(a) and 2(b)). The percent area of the atherosclerotic plaques was measured in both groups as shown in Figure 2(c).
The fibrous cap thickness was measured by drawing lines perpendicular to the lumen at three different locations of the fibrous cap on sections. When compared with that of the normal group, fibrous cap thickness was significantly increased in CIB group $(49.80 \pm 16.96 \mu \mathrm{m}$, Figure 3(a)), and 

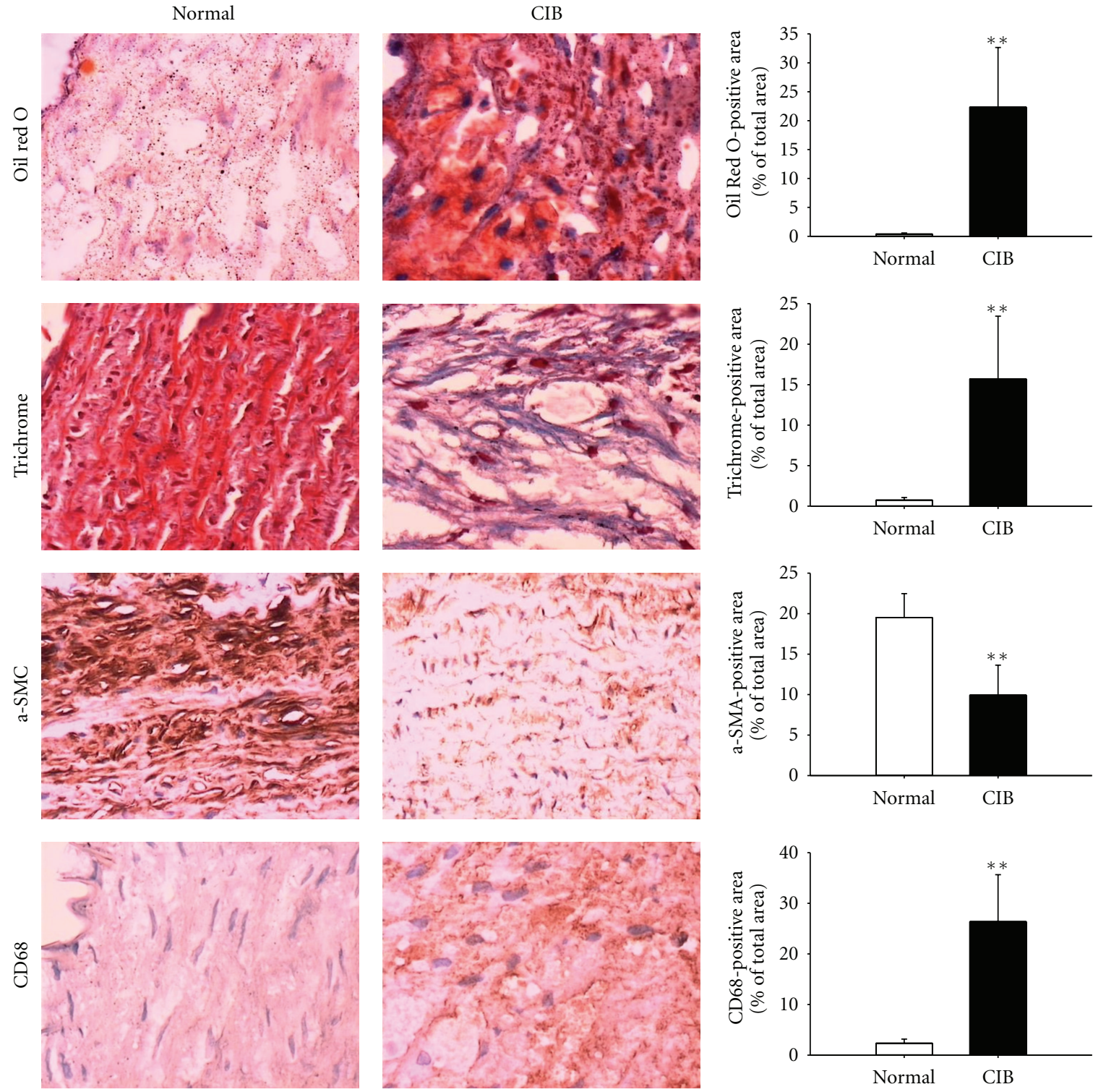

(a)

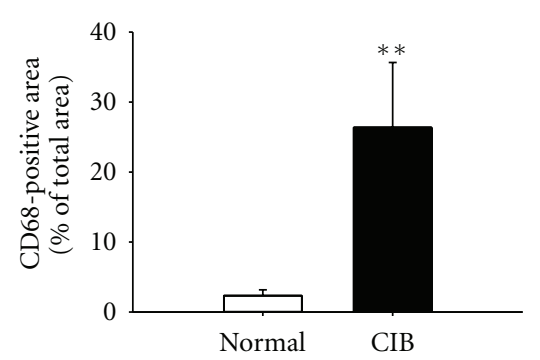

(b)

FIgURE 4: Vulnerability plaque has been identified on CIB rabbit. (a) Representative aortic sections stained by Oil red O, trichrome, CD68 antibody and a SMC-specific antibody (magnification $\times 40$ ). (b) Quantification revealed a significantly larger lipid core, collagen, SMApositive area, and the density of macrophages (identified by CD 68 immunostaining) in CIB rabbits. Data are expressed as mean \pm SD, $* * P<0.01$ compared with normal.

the ratios of fibrous cap and intima media thickness were calculated $(8.52 \pm 4.22$, Figure 3(b)).

The atherosclerotic plaque content of SMCs was lower in the normal group while the content of lipid core area was lower in the CIB group. But CD68 and collagen content of the plaques were higher in the CIB group; as a result, the vulnerability index in the normal group was lower than that in the CIB group (Figure 4(a)). The ratio of lipid core area and plaque area, and ratio of plaque area and intima area were calculated as well (Figure 4(b)).

There was no detectable change in the size of the medial thickness of aortic arch thoracic aorta and abdominal aorta, but the intima thickness of the aorta and the ratio of intima and media in CIB group was the highest. The index of endometrial hyperplasia was remarkably different between the two groups (Figure 5).

4.4. Immunohistochemical Inflammatory Markers and Others. Immunologic injury and high cholesterol control induced a significant increase in systemic inflammatory response in $\mathrm{CIB}$ group. NF- $\kappa \mathrm{B}$ is a key factor that regulates the expression of many genes involved in the pathophysiology of tissue inflammation. In CIB group, NF- $\kappa$ B was increased significantly compared to the normal diet group on the basis of atherosclerotic arteries sections, and strong MMP-9 

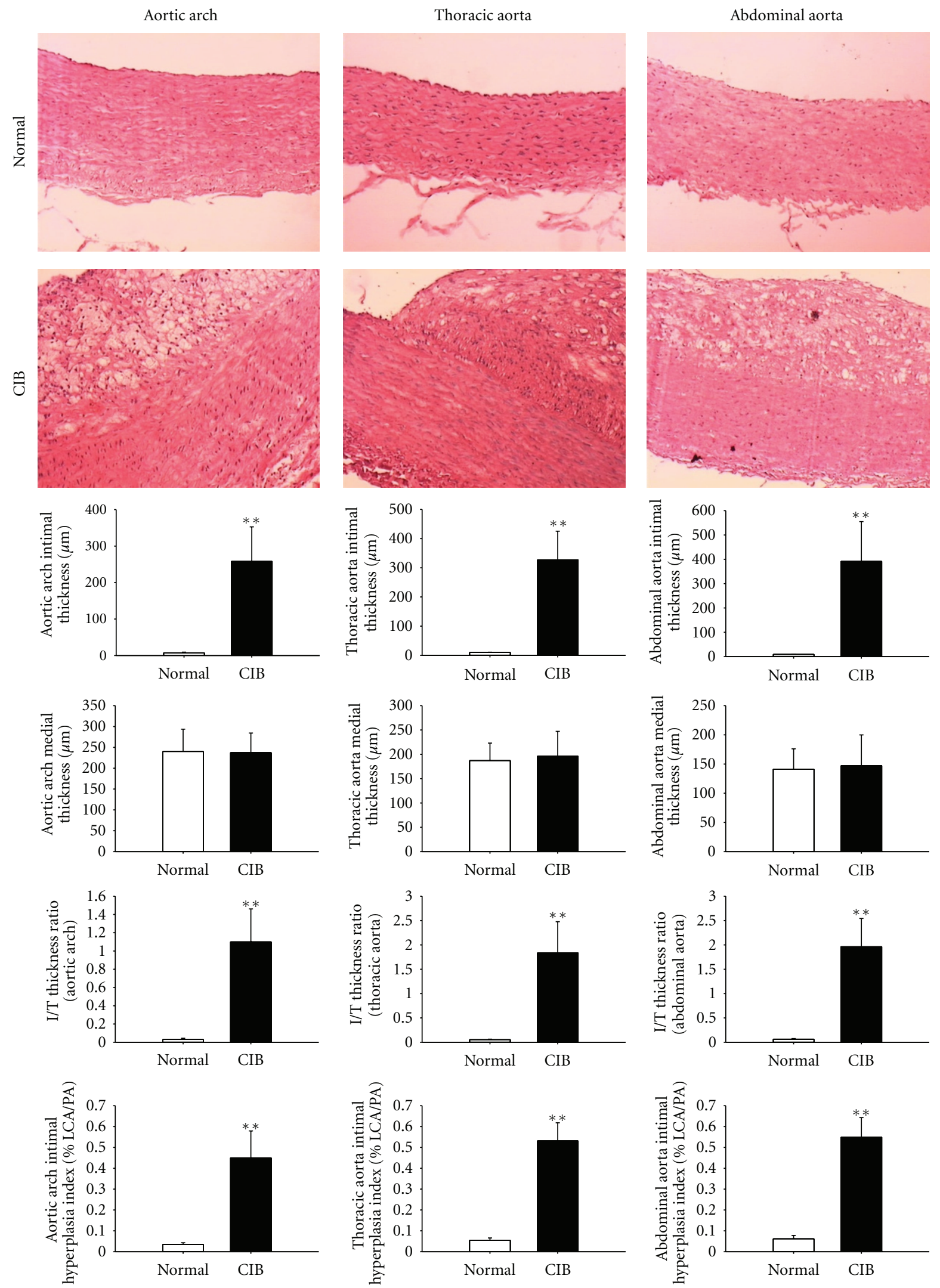

FIGURE 5: Representative hematoxylin-eosin staining for intima/medial thickness of aortic arch, thoracic aorta, and abdominal aorta (magnification $\times 40$ ). The pathology of the abdominal aortic was most serious, followed by the thoracic aorta and aortic arch. Data are expressed as mean $\pm \mathrm{SD}, * P<0.01,{ }^{* *} P<0.01$ compared with normal group. 

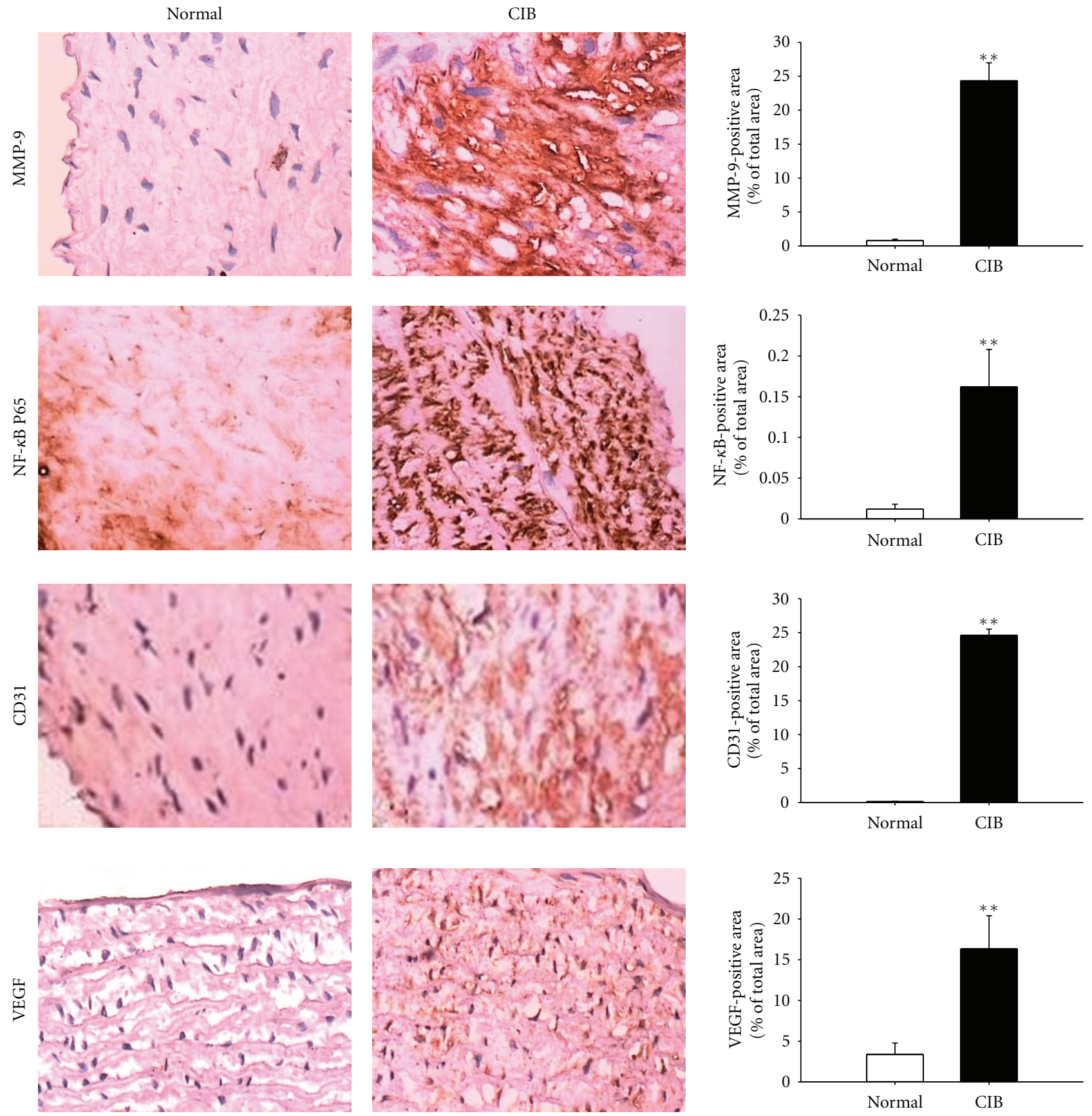

Figure 6: Representative immunohistochemical staining for MMP-9, NF- $\kappa$ B P65 subunit, CD31 and VEGF. Strong positive staining was observed in CIB group and weak staining on the sections of arteries from normal group. MMP-9, matrix metalloproteinase-9; NF- $\kappa \mathrm{B}$ P65 subunit, nuclear factor- $\kappa$ B P65 subunit; CD31, cluster of differentiation 31; VEGF, vascular endothelial growth factor. Data are expressed as mean $\pm \mathrm{SD},{ }^{* *} P<0.01$ compared with normal.

positive staining was also observed And CD31 and VEGF have the same trend with MMP-9 (Figure 6).

4.5. Oxidative Factors. Serum levels of ox-LDL were elevated at week 3 and 6 in the CIB group, but compared to the week 6 , it has a mild reduction. The levels of NO decreased after the immunologic injury, however, at week 10, which was significantly higher in CIB group than in the control group. Serum level of SOD was reduced by the immunologic injury due to the cholesterol diet, but it was elevated at week 6 maybe because the intima was destroyed by balloon injury. Compared to the control group, MDA was increased uniformly at week 3, 6, and 10. In CIB group, MCP-1 was increased significantly compared to the normal diet group at the end of the third week, whereas there was no obvious differences in week 6 . The levels of IL-1 have slightly decreased at week 6 compared to week 3 ; TNF- $\alpha$ kept a same trend with MCP-1 (Figure 7). 

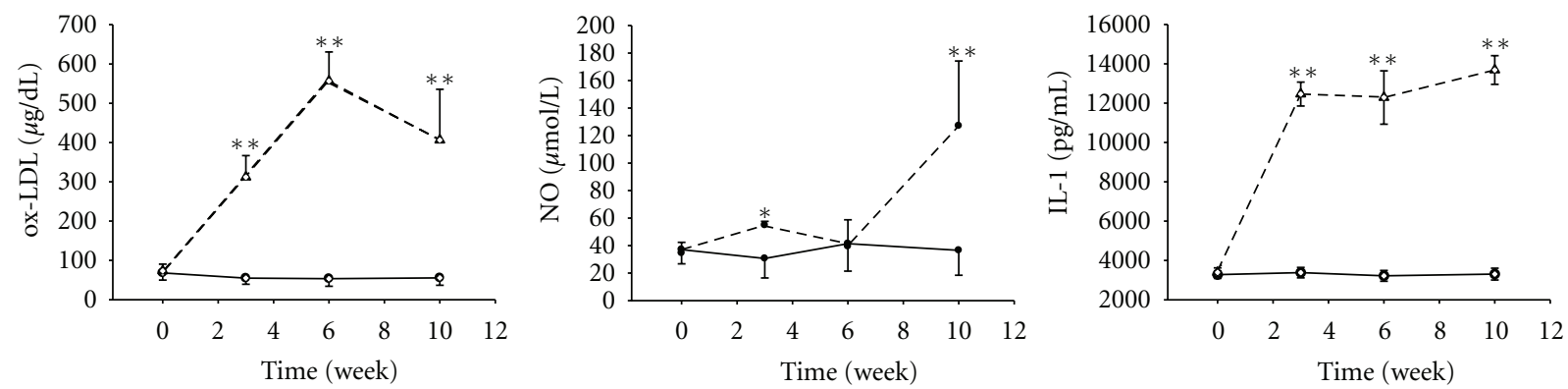

$$
\begin{aligned}
& \multimap-\text { Normal } \\
& -\Delta-\text { CIB }
\end{aligned}
$$

$$
\rightarrow \text { Normal }
$$$$
\rightarrow \text { Normal }
$$$$
-\Delta-\mathrm{CIB}
$$
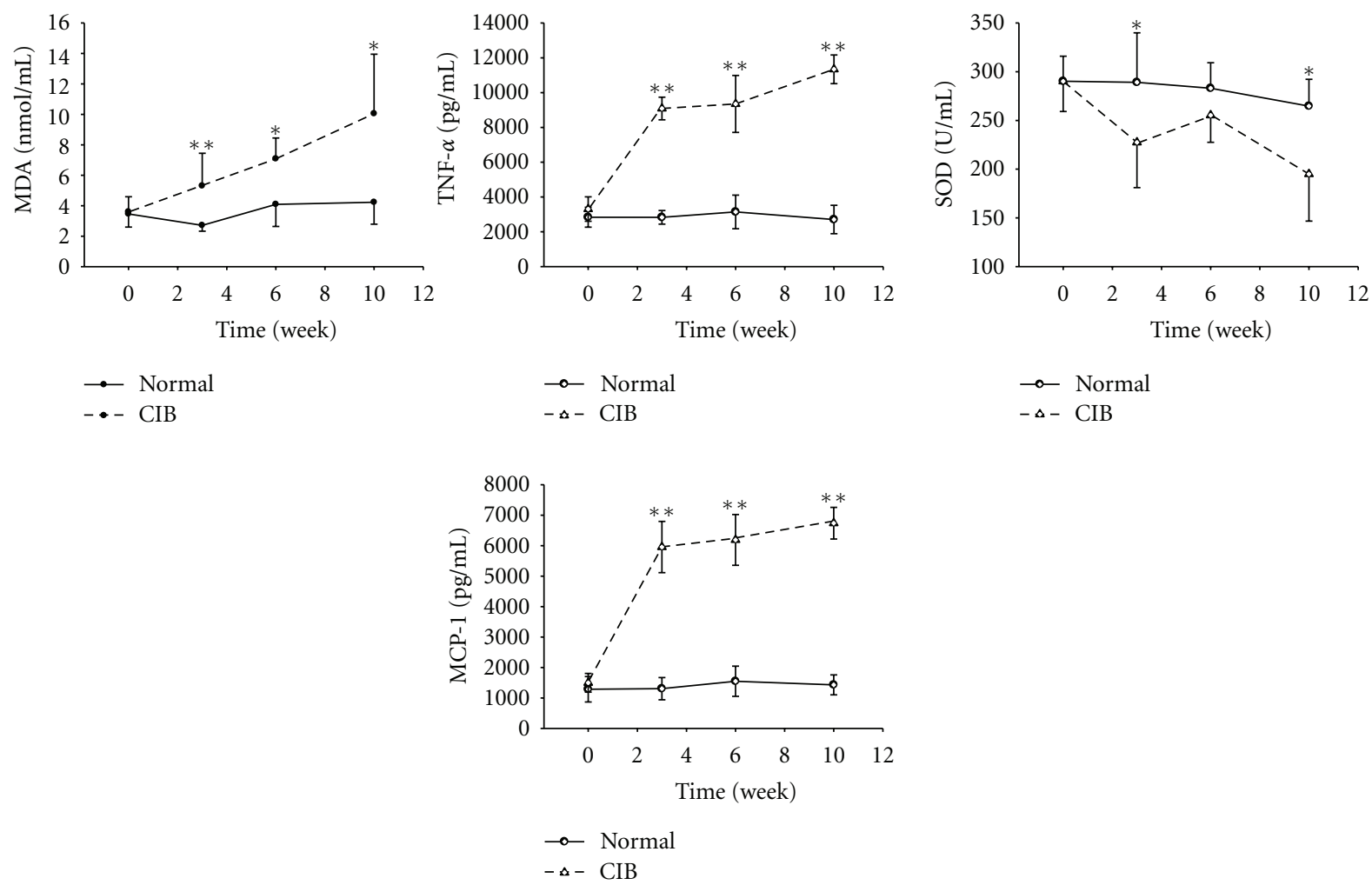

Figure 7: Analysis of biochemical measurements in two groups of rabbits. Serum level of IL-1, ox-LDL, NO, SOD, MDA, MCP-1, and TNF- $\alpha$ by using of enzyme-linked immunosorbent assay on week $0,3,6,10$. IL-1, interleukin-1; ox-LDL, oxidized LDL cholesterol; NO, nitric oxide; SOD, superoxide dismutase; MDA, malondialdehyde; MCP-1, monocyte chemoattractant protein-1; TNF- $\alpha$, tumor necrosis factor- $\alpha$. Data are expressed as mean $\pm \mathrm{SD},{ }^{*} P<0.05,{ }^{* *} P<0.01$ compared with normal group.

\section{Discussion}

Atherosclerosis is characterized by lipid accumulation in the vessel wall, inflammation, oxidative stress, and immunologic injury [23]. There is no perfect animal model that completely replicates all stages of human atherosclerosis, yet the model may be a promising entity in exploring the aetiopathogenesis and regression of atherosclerosis $[24,25]$. In this study, we developed a modified protocol of the JW rabbit that reproduced features of atherothrombosis observed in humans. All the experimental rabbits have been injected with foreign protein, remodeled mechanical endothelial injury, and undergone cholesterol feeding. Histological features of the aorta and the levels of cholesterol, inflammation, and oxidative stress were measured and evaluated to the robust animal models. The key finding of our study is that the rabbit and human atherosclerotic plaques have remarkable similarities. In addition, several novel ideas which enhance our understanding of the mechanisms of human atherosclerotic stages were also introduced by this study.

Cholesterol feeding and mechanical endothelial injury are two common features shared by most models of atherosclerosis [26], but the occurrence of coronary or aortic atherosclerosis in immunopathology factors probably play a major role [27]. We retained the procedure of cholesterol diet for longer durations, which leads to lipid toxicity. And the 
balloon injury could accelerate cholesterol deposition on the arterial wall and injury of the foreign protein maybe make inflammation and oxidative stress of atherosclerosis more serious $[28,29]$.

At the beginning of the experiment, blood lipid distributions of all animals were evaluated and there was no significant difference between the two groups. The highcholesterol diet containing egg yolk and lard oil is very similar to the characteristics of human diet. We chose to perform immunologic injury at two weeks after initiating the high-cholesterol diet, for the immunopathology factors may increase the severity of the local per oxidative damage and inflammatory response [30]. The experimental animals have been injured with balloon at four weeks after initiating the high-cholesterol diet so that endothelial damage occurred in a setting of increased disorders of lipid metabolism, which could further accelerate plaque formation [31]. What makes me surprised was that the serum cholesterol levels were significantly higher in CIB rabbits than in normal diet rabbits at the end of week 3, but not significantly increased after balloon injury comparing to the week 3 . It is becoming increasingly evidential that immunologic injury to the system is probably a primary causative factor in atherosclerosis.

Plaque vulnerability is characterized by a large lipidrich atherosclerotic core, a thin fibrous cap, and infiltration by inflammatory cells, such as macrophages $[32,33]$. Compared with intact caps, the ruptured ones usually are thinner and contain less collagen, have fewer SMCs, and are heavily infiltrated by macrophage foam cells. Atherosclerosis plaques with its constituents, including lipid core structure, proliferating SMCs, and collagen fibers, was observed in the experiment described above. The observations of plaque constituents suggested that there were a higher intima-tomedia thickness ratio and intimal hyperplasia index and were confirmed by previous reports of high macrophage density in areas of plaque rupture $[34,35]$. This correlation proves that the plaque which is characterized by a large lipid-rich athermanous core and a thin fibrous cap is more inflammatory and vulnerable. The experiment also showed that the pathology of the abdominal aortic was most serious, followed by the thoracic aorta and aortic arch. The intima thickness was significantly higher in CIB rabbits than in normal diet rabbits, but not significant different in media thickness between two groups.

Inflammation plays a pivotal role in all stages of thermogenesis, from foam cell to plaque formation to rupture and ultimately to thrombosis, so the inflammatory response in animal blood was evaluated [36, 37]. In this regard, the data suggested that the presence of immunologic injury in highcholesterol diet rabbits results in a more vulnerable vessel wall at the site of the endothelial lesion. IL-6, MCP-1, and TNF- $\alpha$ are three critical mediators of the systemic effects in inflammatory endothelial lesion, and they are also associated with the precipitation of atherosclerotic events. As expected, serum levels of IL-6, MCP-1, and TNF- $\alpha$ increased in the CIB group at week 3, 6, and 10 compared with the control group. The previous studies have demonstrated that MCP-1 expression occurs in the arterial wall in response to hypercholesterolemia in rabbits. Oxidized LDL also induces local vascular cells to produce MCP-1, which causes monocyte recruitment and promotes the release of lipids and liposomal enzymes into the extracellular space, thereby enhancing the progression of the atherosclerotic lesion [38].

It is now recognized that atherosclerotic plaques are characterized not only by the presence of inflammatory reactions but also by oxidative stress. Oxidative stress induces inflammatory responses causing damage to the vasculature and may play an important role in the development of many diseases including atherosclerosis. It also causes direct damage to proteins or leads to chemical modification of amino acids in proteins. This increases the protein content in carbonyls, which then serve as biomarkers of general oxidative stress. The level of per oxidative damage has also been evaluated in this experiment. Oxidized LDL is highly cytotoxic and may promote the development of atherosclerosis by several mechanisms. MDA is a main byproduct of lipid peroxidation; lipid peroxidation is evaluated by levels of circulating MDA in our research. MDA production increases in CIB group early at week 3 , confirming previous reports that an increased formation of lipid peroxidation products occurs. NO is a powerful regulator of vascular function, and it appears that abnormalities in the production or actions of NO lead to endothelial dysfunction and abnormal vascular remodeling. In our experiment, the serum NO level in the CIB group was higher than those in the normal group at the 3rd, but there was a slightly decreased after treating with balloon angioplasty in the 4th week, respectively, and it showed a significant difference at the 10th weekend, which may be that the function of blood vessel endothelium of rabbit was recovered after injuring with balloon angioplasty in the 4 th week.

In conclusion, CIB rabbit, a novel robust model of atherosclerotic plaques, not only has the disorders of lipid metabolism but also carries the metabolic syndrome, which partially caused by immunologic derangement. The pathophysiology of the CIB rabbit coincides well with the current concept of metabolic syndrome, which contends that lipid toxicity accumulation is the fundamental disorder and that inflammation, oxidative stress, and lipid metabolism disorders are intimately related to the evolution of atherosclerosis. As the metabolic pattern for rabbits resembles that in humans, CIB rabbits have superior clinical usefulness compared with other animal models, especially in herbalism fields.

\section{Funding}

This work was supported by the State Key Program of the National Natural Science Foundation of China (no. 30973731; 30901905).

\section{References}

[1] E. Galkina and K. Ley, "Immune and inflammatory mechanisms of atherosclerosis," Annual Review of Immunology, vol. 27, pp. 165-197, 2009. 
[2] A. C. Doran, N. Meller, and C. A. McNamara, "Role of smooth muscle cells in the initiation and early progression of atherosclerosis," Arteriosclerosis, Thrombosis, and Vascular Biology, vol. 28, no. 5, pp. 812-819, 2008.

[3] C. Vinegoni, I. Botnaru, E. Aikawa et al., "Indocyanine green enables near-infrared fluorescence imaging of lipidrich, inflamed atherosclerotic plaques," Science Translational Medicine, vol. 3, no. 84, Article ID 84ra45, 2011.

[4] R. Ross, "Atherosclerosis-an inflammatory disease," The New England Journal of Medicine, vol. 340, no. 2, pp. 115-126, 1999.

[5] G. K. Hansson and P. Libby, "The immune response in atherosclerosis: a double-edged sword," Nature Reviews Immunology, vol. 6, no. 7, pp. 508-519, 2006.

[6] M. Joner, G. Nakazawa, C. Bonsignore et al., "Histopathologic evaluation of nitinol self-expanding stents in an animal model of advanced atherosclerotic lesions," EuroIntervention, vol. 5, no. 6, pp. 737-744, 2010.

[7] M. Sigovan, L. Boussel, A. Sulaiman et al., "Rapid-clearance iron nanoparticles for inflammation imaging of atherosclerotic plaque: initial experience in animal model," Radiology, vol. 252, no. 2, pp. 401-409, 2009.

[8] A. A. Gupte, J. Z. Liu, Y. Ren et al., "Rosiglitazone attenuates age- and diet-associated nonalcoholic steatohepatitis in male low-density lipoprotein receptor knockout mice," Hepatology, vol. 52, no. 6, pp. 2001-2011, 2010.

[9] J. Masuda and R. Ross, "Atherogenesis during low level hypercholesterolemia in the nonhuman primate. II. Fatty streak conversion to fibrous plaque," Arteriosclerosis, vol. 10, no. 2, pp. 178-187, 1990.

[10] D. E. Karangelis, I. Kanakis, A. P. Asimakopoulou et al., "Glycosaminoglycans as key molecules in atherosclerosis: the role of versican and hyaluronan," Current Medicinal Chemistry, vol. 17, no. 33, pp. 4018-4026, 2010.

[11] N. S. van Ditzhuijzen, M. van den Heuvel, O. Sorop et al., "Invasive coronary imaging in animal models of atherosclerosis," Netherlands Heart Journal, vol. 19, no. 10, pp. 442-446, 2011.

[12] J. Nanobashvili, M. Prager, A. Jozkowicz et al., "Positive effect of treatment with synthetic steroid hormone Tibolon on intimal hyperplasia and restenosis after experimental endothelial injury of rabbit carotid artery," European Surgical Research, vol. 36, no. 2, pp. 74-82, 2004.

[13] M. Ragni, P. Golino, P. Cirillo et al., "Endogenous tissue factor pathway inhibitor modulates thrombus formation in an in vivo model of rabbit carotid artery stenosis and endothelial injury," Circulation, vol. 102, no. 1, pp. 113-117, 2000.

[14] O. Lazoura, D. Zacharoulis, T. Kanavou et al., "A novel experimental animal model of arterial stenosis based on endovascular radiofrequency energy application," Journal of Investigative Surgery, vol. 24, no. 3, pp. 123-128, 2011.

[15] L. Zhang, Y. Liu, X. T. Lu et al., "Traditional Chinese medication Tongxinluo dose-dependently enhances stability of vulnerable plaques: a comparison with a high-dose simvastatin therapy," American Journal of Physiology, vol. 297, no. 6, pp. H2004-H2014, 2009.

[16] S. Brizzola, M. De Eguileor, T. Brevini et al., "Morphologic features of biocompatibility and neoangiogenesis onto a biodegradable tracheal prosthesis in an animal model," Interactive Cardiovascular and Thoracic Surgery, vol. 8, no. 6, pp. 610-614, 2009.

[17] F. Acocella, S. Brizzola, C. Valtolina et al., "Prefabricated tracheal prosthesis with partial biodegradable materials: a surgical and tissue engineering evaluation in vivo," Journal of
Biomaterials Science, Polymer Edition, vol. 18, no. 5, pp. 579594, 2007.

[18] C. Calcagno, J. C. Cornily, F. Hyafil et al., "Detection of neovessels in atherosclerotic plaques of rabbits using dynamic contrast enhanced MRI and 18F-FDG PET," Arteriosclerosis, Thrombosis, and Vascular Biology, vol. 28, no. 7, pp. 13111317, 2008.

[19] G. Rega, C. Kaun, S. Demyanets et al., "Vascular endothelial growth factor is induced by the inflammatory cytokines interleukin- 6 and oncostatin $\mathrm{m}$ in human adipose tissue in vitro and in murine adipose tissue in vivo," Arteriosclerosis, Thrombosis, and Vascular Biology, vol. 27, no. 7, pp. 15871595, 2007.

[20] M. Kawasaki, A. Hattori, Y. Ishihara et al., "Tissue characterization of coronary plaques and assessment of thickness of fibrous cap using integrated backscatter intravascular ultrasound-comparison with histology and optical coherence tomography," Circulation Journal, vol. 74, no. 12, pp. 2641-2648, 2010.

[21] Y. Qiao, I. Ronen, J. Viereck, F. L. Ruberg, and J. A. Hamilton, "Identification of atherosclerotic lipid deposits by diffusionweighted imaging," Arteriosclerosis, Thrombosis, and Vascular Biology, vol. 27, no. 6, pp. 1440-1446, 2007.

[22] H. Liu, X. Wang, K. B. Tan et al., "Molecular imaging of vulnerable plaques in rabbits using contrast-enhanced ultrasound targeting to vascular endothelial growth factor receptor-2," Journal of Clinical Ultrasound, vol. 39, no. 2, pp. 83-90, 2011.

[23] J. A. Leopold and J. Loscalzo, "Oxidative risk for atherothrombotic cardiovascular disease," Free Radical Biology and Medicine, vol. 47, no. 12, pp. 1673-1706, 2009.

[24] T. Shimizu, K. Nakai, Y. Morimoto et al., "Simple rabbit model of vulnerable atherosclerotic plaque," Neurologia MedicoChirurgica, vol. 49, no. 8, pp. 327-332, 2009.

[25] L. Xiangdong, L. Yuanwu, Z. Hua, R. Liming, L. Qiuyan, and L. Ning, "Animal models for the atherosclerosis research: a review," Protein and Cell, vol. 2, no. 3, pp. 189-201, 2011.

[26] A. Matsuda, Y. Suzuki, K. Kondo, Y. Ikeda, and K. Umemura, "Hypercholesterolemia induces regression in neointimal thickening due to apoptosis of vascular smooth muscle cells in the hamster endothelial injury model," Cardiovascular Research, vol. 53, no. 2, pp. 512-523, 2002.

[27] P. Krebs, E. Scandella, B. Bolinger, D. Engeler, S. Miller, and B. Ludewig, "Chronic immune reactivity against persisting microbial antigen in the vasculature exacerbates atherosclerotic lesion formation," Arteriosclerosis, Thrombosis, and Vascular Biology, vol. 27, no. 10, pp. 2206-2213, 2007.

[28] A. A. Amran, Z. Zakaria, F. Othman, S. Das, S. Raj L, and N. M. M. Nordin, "Aqueous extract of Piper sarmentosum decreases atherosclerotic lesions in high cholesterolemic experimental rabbits," Lipids in Health and Disease, vol. 9, article 44, 2010.

[29] F. Hakimoğlu, G. Kizil, Z. Kanay, M. Kizil, and H. Isi, "The effect of ethanol extract of Hypericum lysimachioides on lipid profile in hypercholesterolemic rabbits and its in vitro antioxidant activity," Atherosclerosis, vol. 192, no. 1, pp. 113122, 2007.

[30] M. L. Kohut, Y. J. Sim, S. Yu, K. J. Yoon, and C. M. Loiacono, "Chronic exercise reduces illness severity, decreases viral load, and results in greater anti-inflammatory effects than acute exercise during influenza infection," Journal of Infectious Diseases, vol. 200, no. 9, pp. 1434-1442, 2009.

[31] X. Li, J. R. Du, W. D. Wang et al., "Experimental study of effect of tanshinone on artery restenosis in rat carotid injury model," Zhongguo Zhongyao Zazhi, vol. 31, no. 7, pp. 580-584, 2006. 
[32] T. Temma, Y. Ogawa, Y. Kuge et al., “Tissue factor detection for selectively discriminating unstable plaques in an atherosclerotic rabbit model," Journal of Nuclear Medicine, vol. 51, no. 12, pp. 1979-1986, 2010.

[33] H. J. Park, J. Y. Baek, W. S. Shin et al., "Soluble receptor of advanced glycated endproducts is associated with plaque vulnerability in patients with acute myocardial infarction," Circulation Journal, vol. 75, no. 7, pp. 1685-1690, 2011.

[34] T. Gordon, W. P. Castelli, and M. C. Hjortland, "High density lipoprotein as a protective factor against coronary heart disease. The Framingham study," American Journal of Medicine, vol. 62, no. 5, pp. 707-714, 1977.

[35] H. Chen, J. Cai, X. Zhao et al., "Localized measurement of atherosclerotic plaque inflammatory burden with dynamic contrast-enhanced MRI," Magnetic Resonance in Medicine, vol. 64 , no. 2, pp. 567-573, 2010.

[36] R Ross, "Atherosclerosis—an inflammatory disease," The New England Journal of Medicine, vol. 340, no. 2, pp. 115-126, 1999.

[37] J. Fan and T. Watanabe, "Inflammatory reactions in the pathogenesis of atherosclerosis," Journal of Atherosclerosis and Thrombosis, vol. 10, no. 2, pp. 63-71, 2003.

[38] L. Zhong, W. Q. Chen, X. P. Ji et al., "Dominant-negative mutation of monocyte chemoattractant protein-1 prevents vulnerable plaques from rupture in rabbits independent of serum lipid levels," Journal of Cellular and Molecular Medicine, vol. 12, no. 6, pp. 2362-2371, 2008. 

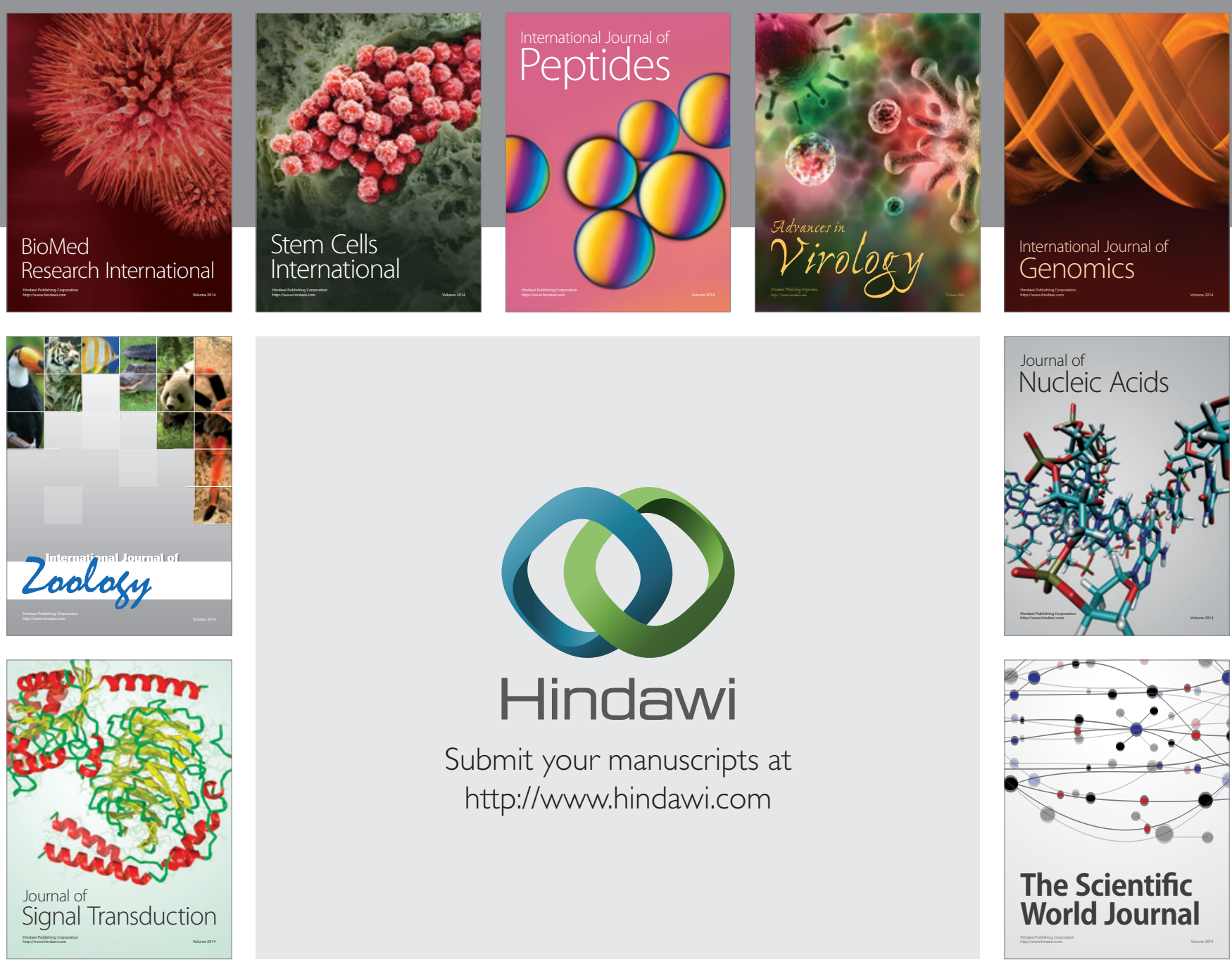

Submit your manuscripts at

http://www.hindawi.com
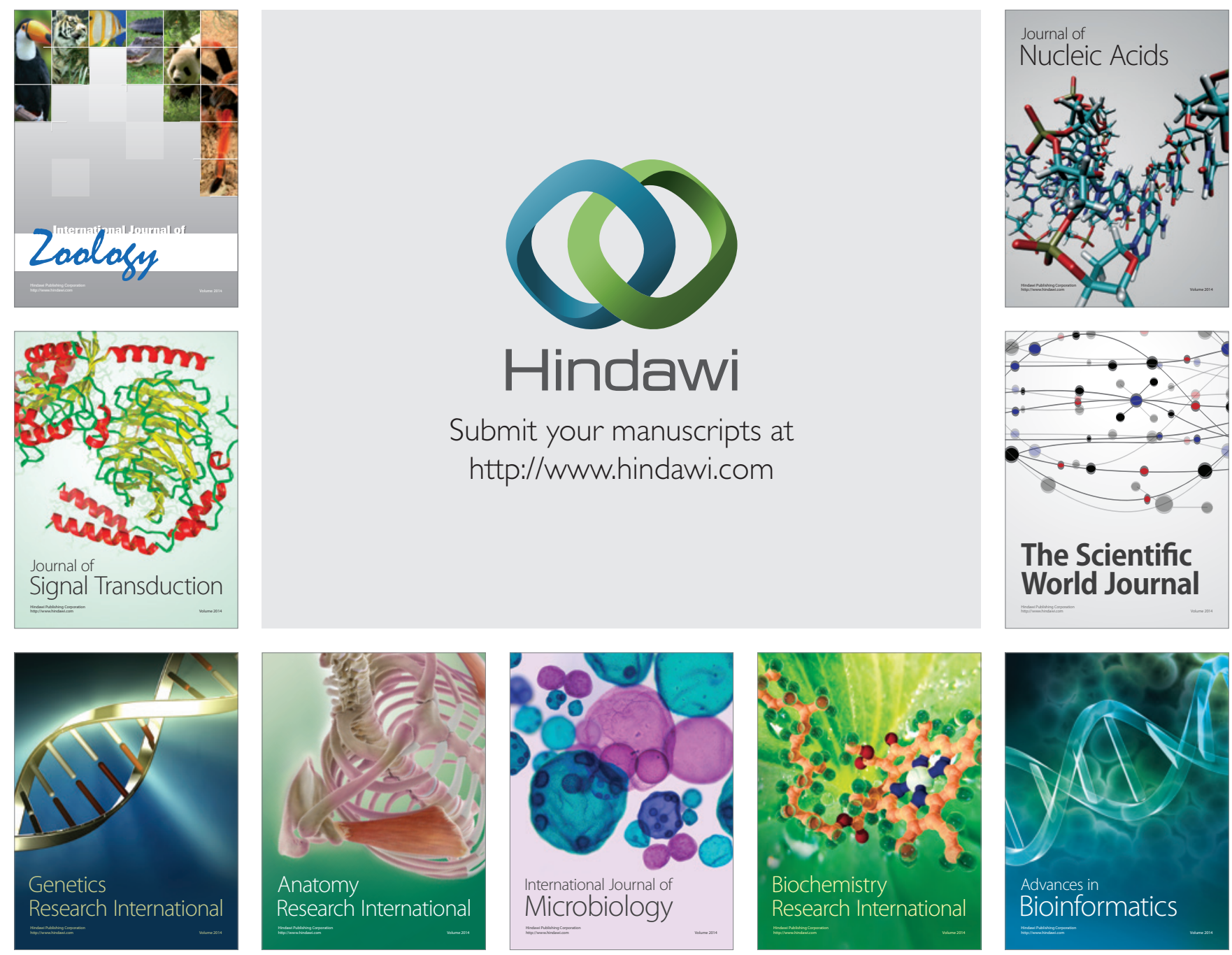

The Scientific World Journal
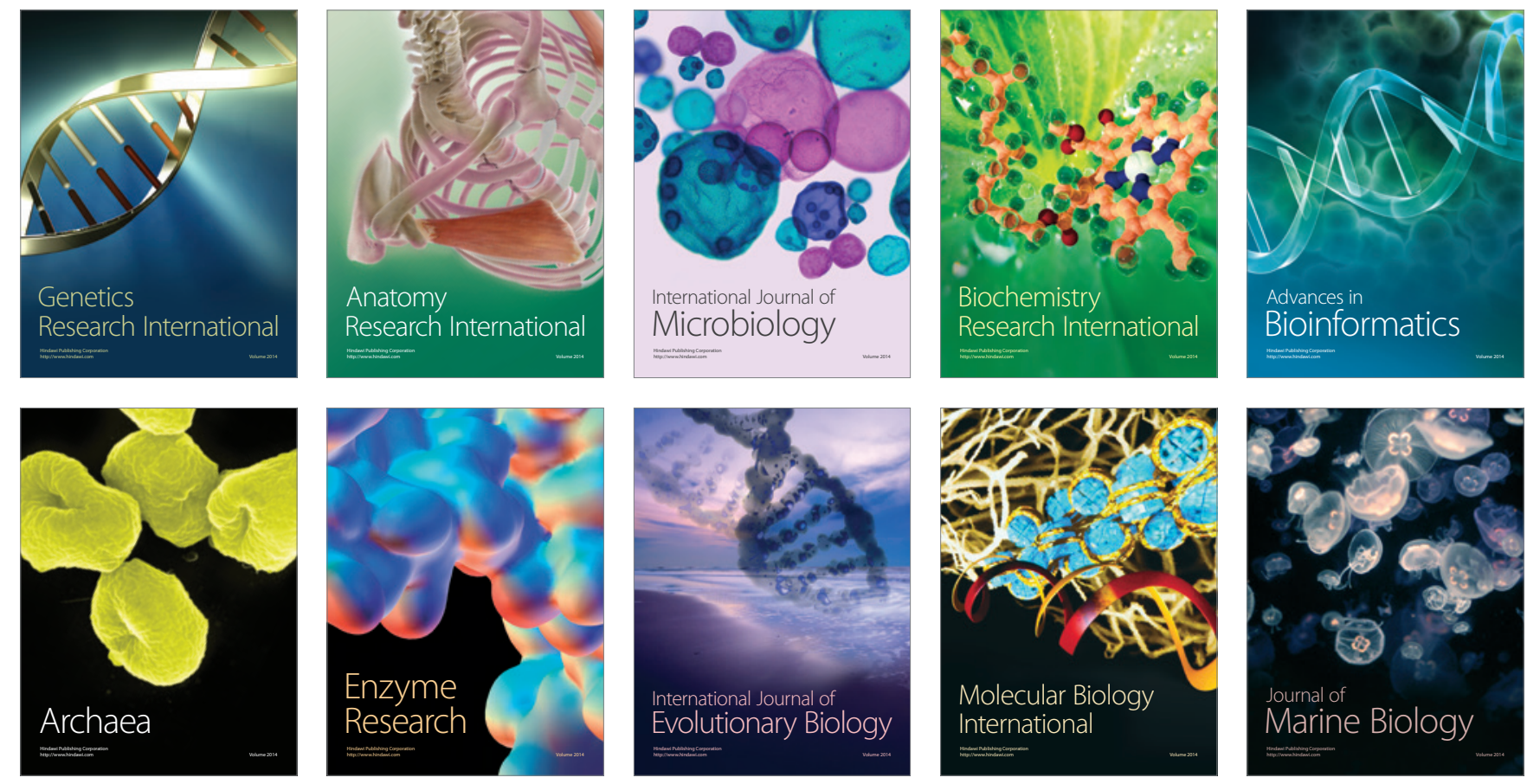